\section{The Unusual Degradation of Recordable Compact Discs}

James Martin, Orion Analytical martin@orionanalvtical.com

The recordable compact disc (CDR) is a popular and inexpensive medium for storing and disseminating digital information, such as micrographs and scanned images. This technical note describes unusual degradation that rendered a group of discs unreadable. The discs were purchased as a set. Each disc was packaged in a jewel box and wrapped in clear plastic. ${ }^{1}$ Several of the discs were used within weeks of purchase to store data, which included digital micrographs. These discs, and the unopened discs, were stored in the dark, in a climatecontrolled room (Temperature: $\cong 20^{\circ} \mathrm{C}$. Relative Humidity: $<55 \%)$.

\section{Degradation}

Within two months of their use, feathery crystals ${ }^{2}$ formed on the surface of the clear polycarbonate substrate (Figure 1). Crystals were subsequently found on several unopened discs. Solubility tests showed that the crystals could be reduced or removed using a soft tissue wetted with ethanol, ${ }^{3}$ but the discs remained unreadable and unwritable.

\section{Analysis}

Microgram surface scrapings of the crystals were analyzed using Fourier transform infrared microspectroscopy. Samples were flattened on diamond cells and analyzed in transmission using a Nicolet Continuum infrared microscope. ${ }^{4}$ Analysis showed that the crystals are bisphenol-A (BPA). ${ }^{5}$

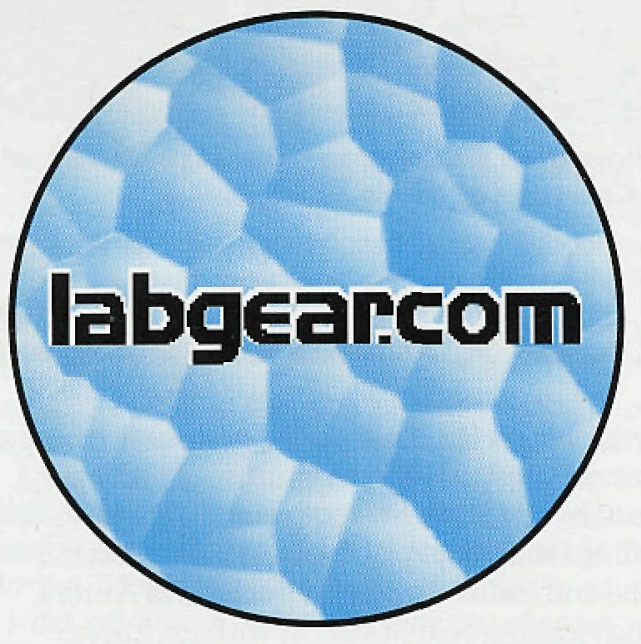

\section{Problem: Find the right equipment,}

\section{... And find it now !! Solution: labgear.com}

- Gather information about microscopy techniques

- Research and find analytical equipment

- Search by technique and then by manufacturer

- Video clips of laboratories and trade shows

- Calendar, new product features, tips from experts

\section{0/20 Foresight Scientific Marketing} $865 / 984-8683$

\section{Source}

The probable source of the BPA is the polycarbonate substrate. Commercial polycarbonates are prepared using BPA. Bisphenol-A is not a component of other layers in a typical CD-R: an organic dye layer, a reflective metallic layer, or a protective lacquer layer.

The actual cause of degradation of the discs has not been established. Experts ${ }^{6}$ consulted for this technical note speculated that the observed degradation resulted from hydrolysis of the polycarbonate layer, which would produce BPA and carbon dioxide. Hydrolysis might occur at elevated temperature and relative humidity ${ }^{7}$ or through contact with a base. ${ }^{8}$ They also cited several cofactors that might contribute to degradation: (1) a high level of residual BPA in the original polymer, (2) impurities in the polycarbonate resin, and (3) improper processing of the disc.

\section{Conclusion}

This unusual form of degradation was unexpected and is, as yet, unexplained. The discs were not exposed to elevated temperature or relative humidity or a base, after purchase. The incident has caused the author to reconsider how and when CD-Rs are used to store digital information. In addition to changing the brand of $C D-R$, multiple copies are made using discs from different manufacturers.

1. The manufacturer will not be named. The color of the lacquered surface is silver.

2. The crystals appeared as dense radiates and arced clusters within the blue dye line and the stacking ring.

3. Alcohols dissolve bisphenol-A, but may induce cracking in the polycarbonate surface that could interfere with data retrieval.

4. Thirty-two sample and background scans were collected at 4 wavenumber resolution using Happ-Genzel apodization.

5. 2,2-bis (4-hydroxyphenol)-propane.

6. The author thanks Chad Mistele (Lexan QO Product Developer, GE Plastics, Mt. Vernon, IN) for sharing his expertise on polycarbonates.

7. Hydrolysis is produced experimentally using a standard hydrolytic test $\left(80^{\circ} \mathrm{C}\right.$ and $\left.85 \% \mathrm{RH}\right)$.

8. Two scenarios were described: (1) contact with a solvent that was slightly basic, such as softened tap water, and (2) in the case of spindled discs, contact with ink from a printed label that contained an organic base.

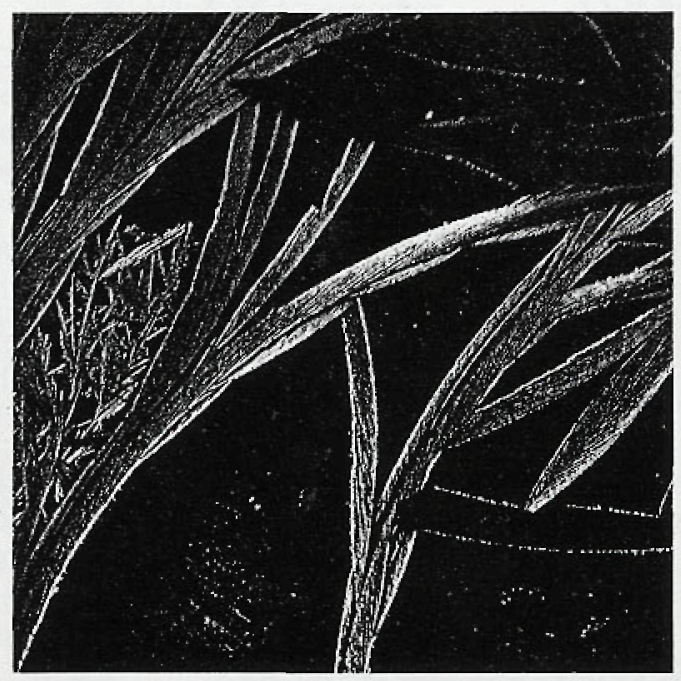

Figure 1: Visible light photomacrograph showing crystals in a $4 \mathrm{~mm}$ square area of a CD-R. 


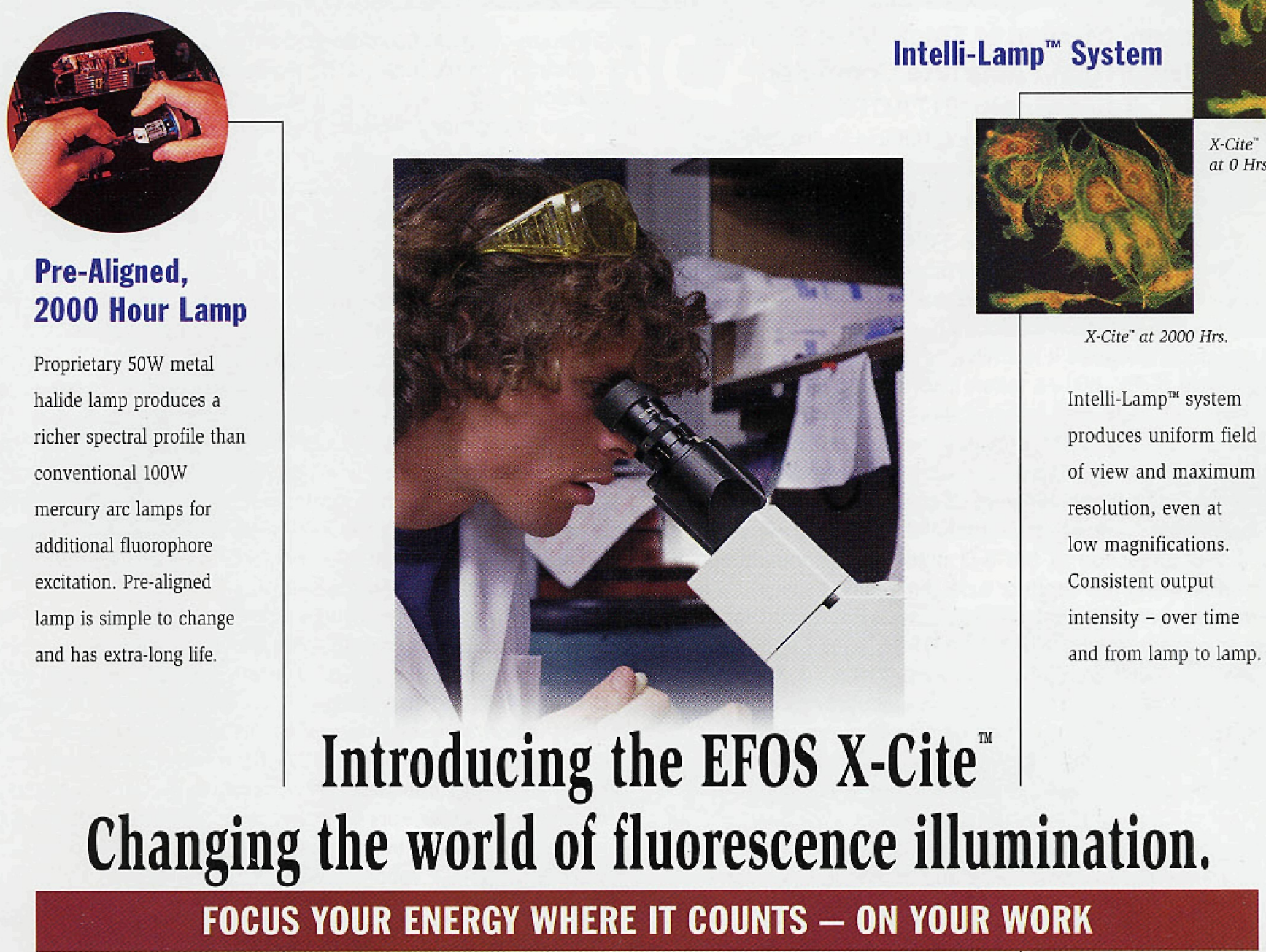

\section{Productivity by Design}

Designed for easier use and better results. Snapon connector makes set-up simple - and you only have to do it once. Remote shutter eliminates vibration. Liquid light guide eliminates sample heating.

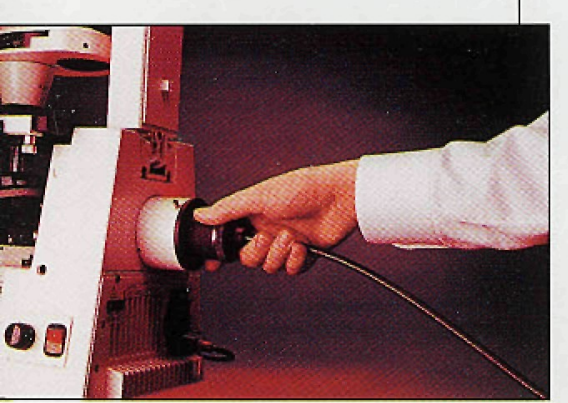

Unique Foot Pedal Control

Leaves hands free for navigation and micromanipulation. Reduces photo-

Brought to you by EFOS, world leaders in light-based technology, the revolutionary new $\mathrm{X}$-Cite ${ }^{\mathrm{Tx}}$ gives you levels of convenience bleaching. and control never before available in a fluorescence illuminator. It's easier to use, less expensive to operate and meets international safety and EMC standards for laboratory equipment. Take a closer look at how the X-Cite ${ }^{\text {Tx }}$ can help you work faster, safer and smarter:

Visit www.efos.com/focus or call 1-800-668-8752.
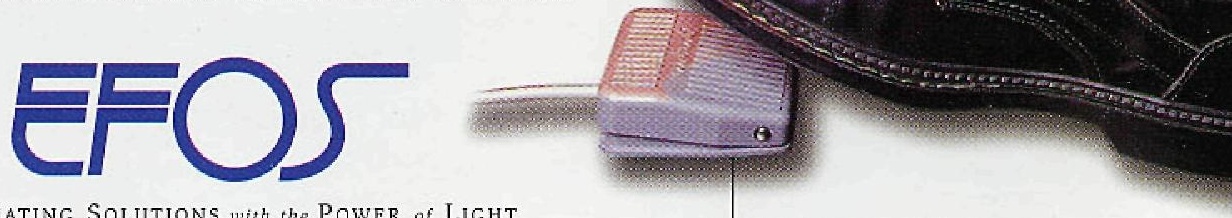